\title{
How do Cosmic Rays enter and leave the Heliosphere?
}

\author{
József Kóta*t \\ The University of Arizona, Tucson AZ 85721-0092, USA \\ E-mail: kota@lpl.arizona.edu
}

\begin{abstract}
The surprisingly abrupt increase of GCRs at the heliopause (HP) seen by both Voyager spacecraft poses a challenge to conventional diffusion models and raises the question how do galactic and anomalous cosmic rays (GCRs and ACRs) enter or leave the heliosphere. In this speculative work we address the possibility that heliospheric currents sheets (HCSs) may provide channels of fast cosmic-ray transport and can act as cracks on the HP. By virtue of fast particle drifts along the HCS, cosmic rays find an easier access to and from the heliosphere at places where a HCS reaches the HP. Numerical simulations adopting a simple toy models is presented and and discussed. The possible dynamical role of GCRs and ACRs on the formation of the HP is briefly addressed. We suggest that the jump in the GCR intensity and the leakage of ACR through the HP might be responsible, in part, for the apparent imbalance between the total pressure inside and outside the HP. Furthermore, the leakage of ACRs might contribute to the flatter than expected size of the inner heliosheath between the termination shock (TS) and HP.
\end{abstract}

36th International Cosmic Ray Conference -ICRC2019-

July 24th - August 1st, 2019

Madison, WI, U.S.A.

* Speaker.

$\dagger$ 


\section{Introduction}

In August, 2012, the Voyager-1 spacecraft crossed the heliopause (HP) and entered into the outer heliosheath (OHS) that is into the interstellar matter flowing around our Heliosphere. Anomalous cosmic rays (ACRs) abruptly disappeared while the intensity of galactic cosmic rays (GCRs) showed an unexpected, almost step-like $\sim 20$ percent increase [1], [2]. This sudden increase of GCRs, which was repeated at the HP crossing of V2 in 2018, is hard to accommodate in the conventional diffusion models that tend to predict more gradual transition through the HP. Numerical models require extremely large parallel and extremely small perpendicular diffusion coefficient to reproduce the observed jump of GCRs [3],[4]. We have casually suggested [3], [5] that besides diffusion, particle drifts may provide channels of preferential transport of cosmic rays to and across the HP. Here, we discuss some implications of this assumption.

The way cosmic rays enter and leave the heliosphere may as well have a role in the dynamics and shape of the HP. Though the pressure of GCRs is quite substantial, its pressure-gradient was thought to be small for the mobile GCRs. This needs to be revisited in the light of the Voyager observations. ACRs leaking through the nose of the HP may also have potential dynamical effects, These are briefly addressed with the purpose of calling attention to possibilities that may deserve further studies.

\section{Can HCS represent cracks on the Heliopause?}

The heliosphere is immersed in a sea of nearly isotropic GCRs. Spatial and temporal intensity variations, with few exceptions (e.g. absorption at the Sun), are connected with with the change of energy of GCRs (measured in the fluid frame) during their propagation in the Heliosphere. Energy change occurs at and inside the termination shock (TS): cosmic rays lose energy inside the TS and have some re-acceleration at the TS The inner heliosheath (IHS between the TS and HP) is a subsonic regime, which can be taken as near incompressible so that the energy change in the IHS can be neglected.

In the absence of energy change, the cosmic ray population between the TS and the HP is a bimodal mixture of pristine interstellar GCRs and GCRs that spent sufficient time to have encountered the TS and change their energy at and inside the TS.

From the Liouville theorem it follows that the phase space density of GCRs consists of $X$ part of GCR spectrum at the TS and $1-X$ part of the pristine interstellar spectrum [6].

$$
f\left(x_{i}, p\right)=X * f_{t s}(p)+(1-X) * f_{i s}(p)
$$

Apart from some rigidity dependence, $X(x, p)$ should be similar for GCRs and ACRs, Hence the time profiles of GCRs and ACRs prior the HP crossing are almost mirror images of each other, whenever GCRs increase ACRs decrease and vice versa.

For a diffusive process we expect $X=0$ at the TS and $X=1$ at the HP, a uniform diffusion results in a linear dependence of $X$ on the distance from the HP. The presence of a HCS may significantly change this simple picture. Earlier we have casually suggested [3],[5] that current sheets (HCSs) may play a role in the transport of ACRs and GCRs through he HP. HCSs do occur close to the HP, sector crossings were observed near the HP [7]. 

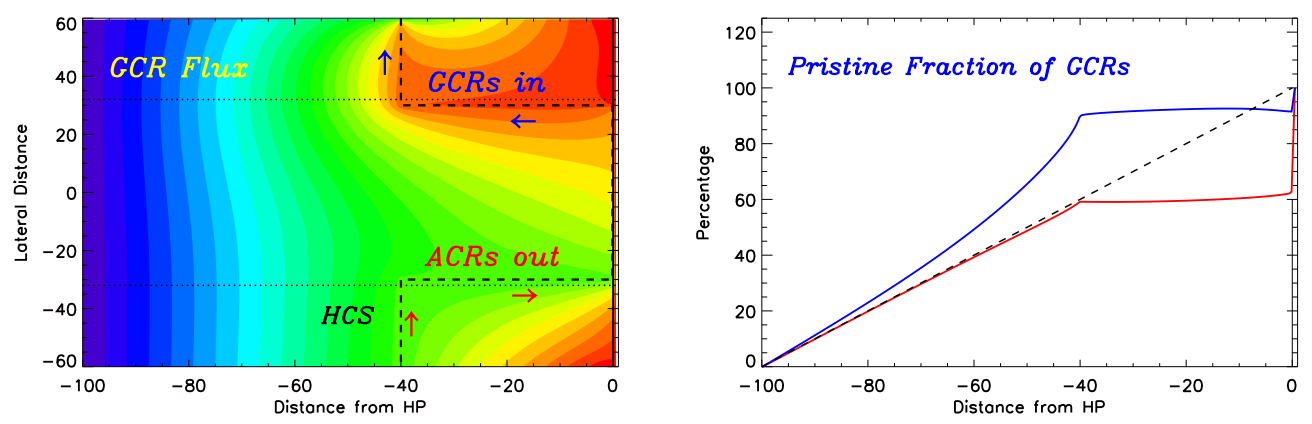

Figure 1: Contour plot of cosmic-ray distribution in a 2-D (x,y) slab containing uniform diffusion $\left(\kappa_{x x}=\kappa_{y y}\right)$ and a current sheet placed along the dashed line (left panel). The left side of the slab $(x=-100)$ plays the role of the TS, while the right side $(x=0)$ plays the role of the HP. The direction of drift is indicated by small arrows. The colors red-to-blue indicate intensities high-to-low. The effect of the HCS is clearly visible (see text). The right panes shows the variation of cosmic-ray flux along the two dotted horizontal lines. An outward HCS drift at and ariund $y=-30$ can lead to a sharp increase of GCRs at the HP.

Figure 1 shows a simple example to visualize the potential effects of HCS drift. Consider a 2-D (x,y) slab with different cosmic ray intensities at the two sides. Assuming an isotropic uniform diffusion would clearly result in a linear dependence of $X$ on the distance from the edges (left TS, $X=0$; right HP, $X=1$ ), shown by the dashed line in the right panel. Now imagine a HCS placed along the dashed line on the left panel, that results in fast particle drifts in the direction indicated by arrows. This drift will assist the inflow of GCRs at one place $(y=+30)$, while will impede the inflow of GCRs at and around $y=-30$. The right panel indicates the variation of $X$ at the dotted horizontal lines at $y=-30$ and $y=+30$. Inspection of Fig. 1 shows that this simple model with a HCS near the HP can lead to a sharp increase of GCRs at the HP, resembling to Voyager observations [1] [2]. ACRs will preferentially leave where drift points outward $(y=-30)$.

Having crossed the HP ACRs quickly move away in a thin belt along the interstellar field lines wrapped around the HP, and they may also drift along the HP, this drift can be considerable if the magnetic field changes substantially at the HP. ACRs seen right beyond the HP by V1 and V2 may have exited several AU (or even tens of AU) from Voyagers. The thickness of the thin belt of ACRs is likely determined by the small random walk of interstellar lines in the OHS. Hence, we can expect very small perpendicular and very large parallel diffusion.

Figure serves demonstration purpose only. Our toy-model is analogous but is not a physical representation of the OHS. We used dimensionless numbers with $\kappa_{x y}^{2}>\kappa_{x x} * \kappa_{y y}$ to make the basic effect more visible. Though the $\kappa_{\perp}$ used here may look small, one should remember that $\kappa_{\perp}$ tends to be small at contact surfaces [8]. A likely reason for this is that the mixing of field lines, which gives the largest contribution to $\kappa_{\perp}$ is inefficient at contact surfaces between two separate fluids.

\section{Dynamical effects of ACRs and GCRs}

Galactic and anomalous cosmic rays may also affect the dynamics and shape of the HP. Comparing the total pressure on the two sides of the HP, Glockler \& Fisk [9] found an apparent imbalance between two sides. The total pressure outside of the HP turned out to be smaller than that 
inside the HP. It is conceivable, that the jump in the GCR pressure, which was not included in the comparison in [9], may account for one part of the missing pressure in the OHS [3]. While GCRs carry substantial pressure, the pressure-gradient had been assumed to be small owing to the high mobility of GCRs. he validity of this assumption needs to revisited $n$ the light of the surprising Voyager observations.

ACRs may have a dynamical role, too. The question is what fraction of ACRs are leaving through the nose section of the HP and what fraction of the ACRs is carried along by the deflected wind down into the heliotail. If all ACRS were convected into to the tail, then a pressure balance should hold at the two sides of the HP. If, on the other hand, a part of ACRs leaks through the HP, then the momentum transport carried by these ACRs can compensate some difference in the respective pressures. ACRs that leave through the HP do quickly move away along the magnetic field lines in a very narrow belt around the HP.

This potential leakage of ACRs through "cracks" on the HP may conceivably also contribute (similarly to a punctured on a tire) to the flatter than expected size of the inner heliosheath (IHS). These ideas need further studies and more careful considerations.

\section{Summary}

in this preliminary report we addressed some questions that may be relevant in understanding the implications of Voayager observations at their HP crossings. We suggested that current sheets that are known to be present near the HP [7] may play a significant role in the transport of GCRs and ACRs. We also suggest that GCRs and ACRs might play significant dynamical role in the formation of the HP: they may be relevant in resolving the apparent pressure-imbalance between the two sides of the HP [9] and the flatter than expected thickness of the IHS. These suggestions are tentative, that need further research.

\section{Acknowledgements}

The author is grateful to J.R. Jokipii, J. Giacalone and F. Fraschetti for valuable discussions.

\section{References}

[1] S.M. Krimigis et al., Science, 341 (2013) 144

[2] E.C. Stone et al., Science, 341 (2013) 150

[3] J. Kóta \& J.R. Jokipii, Astrophys. J., 782 (2014) 24

[4] M. Zhang, X. Luo \& N. Pogorelov, Phys. Plasmas, 22 (2015) id:091501

[5] J. Kóta, JPhCS, 767 (2016) id: 012014

[6] J. Kóta \& J.R. Jokipii, Proc. ICRC, Tsukuba, 3 (2003)?

[7] L.F. Burlaga \& N.F. Ness, Astrophys. J., 784 (2014) 146

[8] D.S. Intriligator et al., J. Geophys. Res., 106 (2001) 10625

[9] G. Gloeckler \& L.A. Fisk Astrophys. J., 833 (2016) 290 